\title{
The coated surface hardness: a kinematic model
}

\author{
J.V. Fernandes ${ }^{a, *}$, L.F. Menezes ${ }^{a}$, A.C. Trindade ${ }^{b}$ \\ ${ }^{a}$ Departamento de Engenharia Mecânica, Faculdade de Ciências e Tecnologia da Universidade de Coimbra, Pinhal de Marrocos-Polo II, 3030 Coimbra, \\ Portugal \\ ${ }^{\mathrm{b}}$ Escola Superior de Tecnologia, Instituto Politécnico de Viseu, 3500Viseu, Portugal
}

Received 23 June 1997; accepted 1 May 1998

\begin{abstract}
A study of the mechanical behaviour of a composite film-substrate material during the hardness test is undertaken. An analytical calculation is proposed applying the kinematic model to the case of the coated spherical cavity problem. The aim of this paper is to discuss the parameters that influence the mechanical behaviour, in order to understand the observed experimental results. The parameters studied are the size of the plastic deformed region, the ratio between the yield stresses of the film and the substrate and the relative work-hardening ratios of both materials. The results show that an important parameter that influences the composite behaviour is the size of the plastic deformed region. The other parameters play an important role in the definition of the size of this region and influence the hardness behaviour as well in a direct way. (C) 1998 Elsevier Science S.A. All rights reserved.
\end{abstract}

Keywords: Modelling; Indentation; Hardness; Monolayers

\section{Introduction}

The indentation test has become the most successful technique to characterise the mechanical behaviour of thin coatings. The main problem associated with this test is the evaluation of the single contributions of the substrate and the thin film in relation to the composite hardness. A large number of theoretical models have been used for this purpose. In spite of their differences, they can be resumed by a general relationship of the form

$\frac{H_{\mathrm{c}}-H_{\mathrm{s}}}{H_{\mathrm{f}}-H_{\mathrm{s}}}=F\left(\frac{t}{h}\right)$

where $H_{\mathrm{c}}, H_{\mathrm{s}}$ and $H_{\mathrm{f}}$ are the composite, substrate and film hardnesses, respectively, $t$ is the film thickness and $h$ is the depth of the indentation. The function $F$ depends on the type of model and could have a more or less complex form [112]. Most of the times, $F$ is a polynomial function with a degree equal to or inferior than 3 and the respective constants must depend on parameters related with the properties of the film and the substrate materials, size and geometry of the deformed region, etc. This dependence is not well understood and, in general, it is taken in an empirical way.

In another paper [13], the authors develop an empirical

\footnotetext{
* Corresponding author. Tel.: +351 39 790700; fax: +351 39790701 .
}

model to predict the hardness of the film from the measured hardness of a film-substrate bilayer. This empirical model, that can be used when the maximum load is such that the plastic deformed region clearly affects the substrate, is based on results obtained using depth sensing hardness instruments. From the studied experimental cases, it was understood that the size of the deformed region, more precisely, the ratio of the film thickness/critical depth (above which exists plastic deformation of the substrate) is an important parameter to determine the constants of the model.

The intention of this paper is to undertake a theoretical study of the mechanical behaviour of a bilayer, in order to model the hardness test applying the kinematic approach to the case of the coated spherical cavity problem. The kinematic model assumes that the plastic work done by the stress field on a kinematically admissible displacement increment field is equal to the work done by the external loads on the same displacement increment field. The main objective of the present study is to appreciate the influence of several parameters, concerning the size of the deformed region and the mechanical behaviours of the film and the substrate, on the relationship given by Eq. (1). The present manipulation of the kinematic approach assume the hypotheses that the mechanical behaviour of both film and substrate are rigidplastic with linear work-hardening. Indeed, as most materials present ductile behaviour, specially under compression 
stress state, as in the case of the hardness test, the rigidplastic theory can then be applied. This formulation discards the elastic part of the deformation, which is quite reasonable when the plastic strain reaches large values compared with the elastic strain values.

\section{A kinematics model analysis of a coated spherical cavity problem}

The kinematics model defines an admissible displacement increment field $\mathrm{d} u_{i}^{*}$ that satisfies the conditions of strain compatibility, plastic incompressibility and the boundary conditions. The plastic work done by the stress field $W_{\mathrm{i}}$ on a kinematically admissible displacement increment field is an upper bound of the work done by the external loads $W_{\mathrm{e}}$ on the same displacement field [14-17]. So, the upper bound of external load per unity of area $T_{1}$ can be obtained solving the equation

$W_{\mathrm{e}}=\int_{S} T_{i} \mathrm{~d} u_{i} \mathrm{~d} S=\int_{V} \sigma_{i j}^{*} \mathrm{~d} \varepsilon_{i j}^{*} \mathrm{~d} V=W_{\mathrm{i}}$

where $\mathrm{d} u_{i}$ represents the boundary displacement, $S$ the boundary surface, $\mathrm{d} \varepsilon_{i j}^{*}$ the kinematically admissible incremental strain field, $\sigma_{i j}^{*}$ the stress tensor at each point and $V$ the plastically deformed volume. The physical meaning of this equation is as follows: for all possible kinematically admissible displacement fields, the work done by the associated stress field in the volume is equal to the work done by the unknown forces during the displacement of the surface boundary.

Both sides of the above equation can be divided by $\mathrm{d} t$ and the displacement increment field becomes a velocity field

$\dot{W}_{\mathrm{e}}=\int_{S} T_{i} v_{i} \mathrm{~d} S=\int_{V} \sigma_{i j}^{*} \dot{\varepsilon}_{i j}^{*} \mathrm{~d} V=\dot{W}_{\mathrm{i}}$

where $\nu_{i}$ is the boundary velocity and $\dot{\varepsilon}_{i j}^{*}$ represents the components of the strain rate tensor.

The hardness of a material depends on several parameters such as the yield stresses $\sigma_{0}$, the Young's modulus $E$ and the flow rule (plastic stress-strain relation) [18-20]. For small ratios $\sigma_{0} / E$ the elastic behaviour does not influence the plastic flow under the indent. So, the hardness can be calculated using a rigid-plastic behaviour model [20].

Considering that a pressured spherical cavity, existing within a rigid-plastic bulk material, has radius $a$ and is submitted to a pressure $p$, the incremental displacements can be written using spherical co-ordinates as follows

$\mathrm{d} u_{\theta}=\mathrm{d} u_{\varphi}=0$

$\mathrm{d} u_{r}=\mathrm{d} u(r)$

and the components of the incremental plastic deformation tensor are

$\mathrm{d} \varepsilon_{r r}=\frac{\mathrm{d}}{\mathrm{d} r} \mathrm{~d} u(r)$ $\mathrm{d} \varepsilon_{\theta \theta}=\mathrm{d} \varepsilon_{\varphi \varphi}=\frac{\mathrm{d} u(r)}{r}$

$\mathrm{d} \varepsilon_{\theta \varphi}=\mathrm{d} \varepsilon_{r \theta}=\mathrm{d} \varepsilon_{r \varphi}=0$

The incompressibility condition gives

$\mathrm{d} \varepsilon_{r r}+\mathrm{d} \varepsilon_{\theta \theta}+\mathrm{d} \varepsilon_{\varphi \varphi}=0 \Rightarrow \mathrm{d} \varepsilon_{r r}+2 \mathrm{~d} \varepsilon_{\theta \theta}=0$

Taking into consideration the boundary condition that at $r=a$ (a is the inner radius of the spherical cavity), $\mathrm{d} u$ is equal to $\mathrm{d} a$, the solution of this differential equation is

$\mathrm{d} u=\frac{a^{2}}{r^{2}} \mathrm{~d} a$

So, Eq. (5) can be written as follows

$\mathrm{d} \varepsilon_{r r}=-\frac{2 a^{2}}{r^{3}} \mathrm{~d} a$

$\mathrm{d} \varepsilon_{\theta \theta}=\mathrm{d} \varepsilon_{\varphi \varphi}=\frac{a^{2}}{r^{3}} \mathrm{~d} a$

$\mathrm{d} \varepsilon_{\theta \varphi}=\mathrm{d} \varepsilon_{r \theta}=\mathrm{d} \varepsilon_{r \varphi}=0$

or, dividing both members of the above equation by the time increment $\mathrm{d} t$, and doing $\dot{\varepsilon}_{i j}=\left(\mathrm{d} \varepsilon_{i j} / \mathrm{d} t\right)$ and $\dot{a}=(\mathrm{d} a / \mathrm{d} t)$

$\dot{\varepsilon}_{r r}=-\frac{2 a^{2}}{r^{3}} \dot{a}$

$\dot{\varepsilon}_{\theta \theta}=\dot{\varepsilon}_{\varphi \varphi}=\frac{a^{2}}{r^{3}} \dot{a}$

$\dot{\varepsilon}_{\theta \varphi}=\dot{\varepsilon}_{r \theta}=\dot{\varepsilon}_{r \varphi}=0$

Considering this equation, the definition of the von Mises equivalent plastic strain rate gives

$$
\begin{aligned}
\dot{\bar{\varepsilon}} & =\left(\frac{2}{\sqrt{3}}\right) \sqrt{\left(\frac{1}{2} \sum \dot{\varepsilon}_{i j} \dot{\varepsilon}_{i j}\right)}=\left(\sqrt{\frac{2}{3}}\right) \sqrt{\dot{\varepsilon}_{r r}^{2}+\dot{\varepsilon}_{\theta \theta}^{2}+\dot{\varepsilon}_{\varphi \varphi}^{2}} \\
& =2 \frac{a^{2}}{r^{3}} \dot{a}
\end{aligned}
$$

A linear work-hardening can be considered in the following way

$\bar{\sigma}=\sigma_{0}+\alpha \bar{\varepsilon}$

where $\bar{\sigma}$ and $\bar{\varepsilon}$ are the equivalent stress and plastic strain after von Mises. From Eq. (9) and considering that $\varepsilon_{r r}=0$ when $r=c$ (in the elastoplastic boundary), it is possible to write

$\varepsilon_{r r}=-\frac{2}{3}\left(\frac{a}{c}\right)^{3}\left(\frac{c^{3}}{r^{3}}-1\right)$ 


$$
\begin{aligned}
\bar{\varepsilon} & =\left(\frac{2}{\sqrt{3}}\right) \sqrt{\left(\frac{1}{2} \sum \varepsilon_{i j} \varepsilon_{i j}\right)}=\left(\sqrt{\frac{2}{3}}\right) \sqrt{\varepsilon_{r r}^{2}+\varepsilon_{\theta \theta}^{2}+\varepsilon_{\varphi \varphi}^{2}} \\
& =\frac{2}{3}\left(\frac{a}{c}\right)^{3}\left(\frac{c^{3}}{r^{3}}-1\right) \\
\bar{\sigma} & =\sigma_{0}+\frac{2}{3} \alpha\left(\frac{a}{c}\right)^{3}\left(\frac{c^{3}}{r^{3}}-1\right)
\end{aligned}
$$

The internal plastic energy rate $\dot{W}_{\mathrm{i}}$ can be calculated considering that in Eq. (3) $\dot{\sigma}_{i j}^{*} \dot{\varepsilon}_{i j}^{*}$ can be substituted by $\bar{\sigma}^{*} \dot{\bar{\varepsilon}}^{*}$, being $\bar{\sigma}^{*}$ and $\dot{\bar{\varepsilon}}^{*}$ the equivalent stress and plastic strain rate after von Mises, as follows

$$
\begin{aligned}
\dot{W}_{\mathrm{i}} & =\int_{V} \bar{\sigma}^{*} \dot{\bar{\varepsilon}}^{*} \mathrm{~d} V \\
& =4 \pi a^{2} \dot{a}\left\{\sigma_{0} \ln \left(\frac{c}{a}\right)+\frac{2}{9} \alpha\left[1-\left(\frac{a}{c}\right)^{3}\left(1+3 \ln \left(\frac{c}{a}\right)\right)\right]\right\}
\end{aligned}
$$

The energy rate of external forces along the displacement boundaries $\dot{W}_{\mathrm{e}}$, can be calculated as follows

$\dot{W}_{\mathrm{e}}=\int_{S} T_{i} v_{i} \mathrm{~d} S=2 \pi a^{2} p \dot{a}$

The Eq. (3) then allows us to write

$$
H V=p=2 \sigma_{0} \ln \left(\frac{c}{a}\right)+\frac{4}{9} \alpha\left\{1-\left(\frac{a}{c}\right)^{3}\left[1+3 \ln \left(\frac{c}{a}\right)\right]\right\}
$$

Considering that $(a / c)^{3}[1+3 \ln (c / a)]$ can be neglected when compared with the unity ( $c$ is about five times $a$ and so $(a / c)^{3} \approx 0$ ), Eq. (17) can be written as follows

$H V=p=2 \sigma_{0} \ln \left(\frac{c}{a}\right)+\frac{4}{9} \alpha=2 \sigma_{0}\left[\ln \left(\frac{c}{a}\right)+\frac{2}{9}\left(\frac{\alpha}{\sigma_{0}}\right)\right]$

If the material does not present work-hardening $(\alpha=0)$ this equation is reduced to

$H V=p=2 \sigma_{0} \ln \left(\frac{c}{a}\right)$

In the case of a composite material, the internal work $\dot{W}_{\mathrm{i}}$ is given by

$\dot{W}_{\mathrm{i}}=\int_{V} \bar{\sigma}^{*} \dot{\bar{\varepsilon}}^{*} \mathrm{~d} V=\int_{V_{\mathrm{f}}} \bar{\sigma}^{*} \dot{\bar{\varepsilon}}^{*} \mathrm{~d} V_{\mathrm{f}}+\int_{V_{\mathrm{s}}} \bar{\sigma}^{*} \dot{\bar{\varepsilon}}^{*} \mathrm{~d} V_{\mathrm{s}}$

where $V_{\mathrm{f}}$ and $V_{\mathrm{s}}$ are the plastic deformed volume of the film and substrate, respectively. We consider an identical behaviour for the film and substrate as that described by Eq. (11) $\bar{\sigma}=\sigma_{0}+\alpha_{\mathrm{s}} \bar{\varepsilon}$

$\bar{\Sigma}=\Sigma_{0}+\alpha_{\mathrm{f}} \bar{\varepsilon}$

where $\alpha_{\mathrm{s}}$ and $\alpha_{\mathrm{f}}, \sigma_{0}$ and $\Sigma_{0}, \bar{\sigma}$ and $\bar{\Sigma}$ are the workhardening linear coefficients, the yield stresses and the equivalent stresses for the substrate and for the film, respectively.

The internal plastic energy rate $\dot{W}_{i}$ can now be calculated as follows

$$
\begin{aligned}
\dot{W} \mathrm{i}= & 4 \pi a^{2} \dot{a}\left\{\int_{a}^{a+t}\left[\Sigma_{0}+\left(\frac{2}{3}\right) \alpha_{f}\left(\frac{a}{c}\right)^{3}\left(\frac{c^{3}}{r^{3}}-1\right)\right] \frac{1}{r} \mathrm{~d} r\right. \\
& \left.+\int_{a+t}^{c}\left[\sigma_{0}+\left(\frac{2}{3}\right) \alpha_{\mathrm{s}}\left(\frac{a}{c}\right)^{3}\left(\frac{c^{3}}{r^{3}}-1\right)\right] \frac{1}{r} \mathrm{~d} r\right\}
\end{aligned}
$$

If we suppose the following relationship between the characteristics of the film and the substrate to exist

$\Sigma_{0}=k \sigma_{0}$

$\alpha_{\mathrm{f}}=x \alpha_{\mathrm{s}}$

then, it is possible to write

$$
\begin{aligned}
\dot{W}_{\mathrm{i}}= & 4 \pi a^{2} \dot{a}\left\{\sigma_{0} \ln \left[\left(\frac{a+t}{a}\right)^{k-1} \frac{c}{a}\right]+\frac{2}{9} \alpha_{\mathrm{s}}\left[x-\left(\frac{a}{c}\right)^{3}\right]\right. \\
& +\frac{2}{9} \alpha_{\mathrm{s}}\left(\frac{a}{a+t}\right)^{3}(1-x)-\frac{2}{3} \alpha_{\mathrm{s}}\left(\frac{a}{c}\right)^{3} \\
& \left.\times \ln \left[\left(\frac{a+t}{a}\right)^{x-1} \frac{c}{a}\right]\right\}
\end{aligned}
$$

The energy rate of external forces along the displacement boundaries $\dot{W}_{\mathrm{e}}$ has the same value as in Eq. (16). So, Eq. (3) allows us to write

$$
\begin{aligned}
H_{\mathrm{c}}=p= & 2\left\{\sigma_{0} \ln \left[\left(\frac{a+t}{a}\right)^{k-1} \frac{c}{a}\right]+\frac{2}{9} \alpha_{\mathrm{s}}\left[x-\left(\frac{a}{c}\right)^{3}\right]\right. \\
& +\frac{2}{9} \alpha_{\mathrm{s}}\left(\frac{a}{a+t}\right)^{3}(1-x)-\frac{2}{3} \alpha_{\mathrm{s}}\left(\frac{a}{c}\right)^{3} \\
& \left.\times \ln \left[\left(\frac{a+t}{a}\right)^{x-1} \frac{c}{a}\right]\right\}
\end{aligned}
$$

Considering that $H_{\mathrm{s}}$ can be calculated using Eq. (17) and that the value of $(a / c)$ is the same for the substrate, the film and the composite, it is possible to deduce the following expression 


$$
\begin{aligned}
\frac{H_{\mathrm{c}}-H_{\mathrm{s}}}{H_{\mathrm{f}}-H_{\mathrm{s}}}= & \left\{\left[1+\frac{2}{3} \frac{\left(\frac{\alpha_{s}}{\sigma_{0}}\right)}{(k-1)}\left(\frac{a}{c}\right)^{3}(1-x)\right] \ln \left(1+\frac{t}{a}\right)\right. \\
& \left.-\frac{2}{9} \frac{\left(\frac{\alpha_{s}}{\sigma_{0}}\right)}{(k-1)}(1-x)\left[1-\left(1+\frac{t}{a}\right)^{-3}\right]\right\} \\
& \div\left\{\left[1+\frac{2}{3} \frac{\left(\frac{\alpha_{s}}{\sigma_{0}}\right)}{(k-1)}\left(\frac{a}{c}\right)^{3}(1-x)\right]\right. \\
& \left.\times \ln \left(\frac{c}{a}\right)-\frac{2}{9} \frac{\left(\frac{\alpha_{s}}{\sigma_{0}}\right)}{(k-1)}(1-x)\left[1-\left(\frac{a}{c}\right)^{3}\right]\right\}
\end{aligned}
$$

Assuming $(a / c)^{3} \approx 0$ and considering the equality $\left(\alpha_{\mathrm{s}} / \sigma_{0}\right) /(k-1)=\alpha_{\mathrm{s}} /\left(\Sigma_{0}-\sigma_{0}\right)$, this equation becomes

$\frac{H_{\mathrm{c}}-H_{\mathrm{s}}}{H_{\mathrm{f}}-H_{\mathrm{s}}}=$

$$
\frac{\ln \left(1+\frac{t}{a}\right)-\frac{2}{9}\left(\frac{\alpha_{s}}{\Sigma_{0}-\sigma_{0}}\right)(1-x)\left[1-\left(1+\frac{t}{a}\right)^{-3}\right]}{\ln \left(\frac{c}{a}\right)-\frac{2}{9}\left(\frac{\alpha_{s}}{\Sigma_{0}-\sigma_{0}}\right)(1-x)}
$$

If both materials do not present work-hardening, i.e. neglecting $\alpha_{\mathrm{s}}$ and $\alpha_{\mathrm{f}}$, this equation is reduced to

$\frac{H_{\mathrm{c}}-H_{\mathrm{s}}}{H_{\mathrm{f}}-H_{\mathrm{s}}}=\frac{\ln \left(1+\frac{t}{a}\right)}{\ln \left(\frac{c}{a}\right)}$

Taking into account Eq. (19), the above equation can also be formulated as follows

$\frac{H_{\mathrm{c}}-H_{\mathrm{s}}}{H_{\mathrm{f}}-H_{\mathrm{s}}}=\frac{\ln \left(1+\frac{t}{a}\right)}{\frac{H_{\mathrm{s}}}{2 \sigma_{0}}}$

This result makes it possible to plot $\left(H_{\mathrm{c}}-H_{\mathrm{s}}\right) /\left(H_{\mathrm{f}}-H_{\mathrm{s}}\right)$ versus $(t / a)$, knowing the ratio between the hardness and the yield stress of the substrate $\left(\left(H \mathrm{~s} / \sigma_{0}\right) \approx 3\right.$ as discussed below). In this simplified formulation, the present model is identical to the one proposed by Ford [8].

\section{Discussion of the model results}

When applying the cavity approach to model the beha-

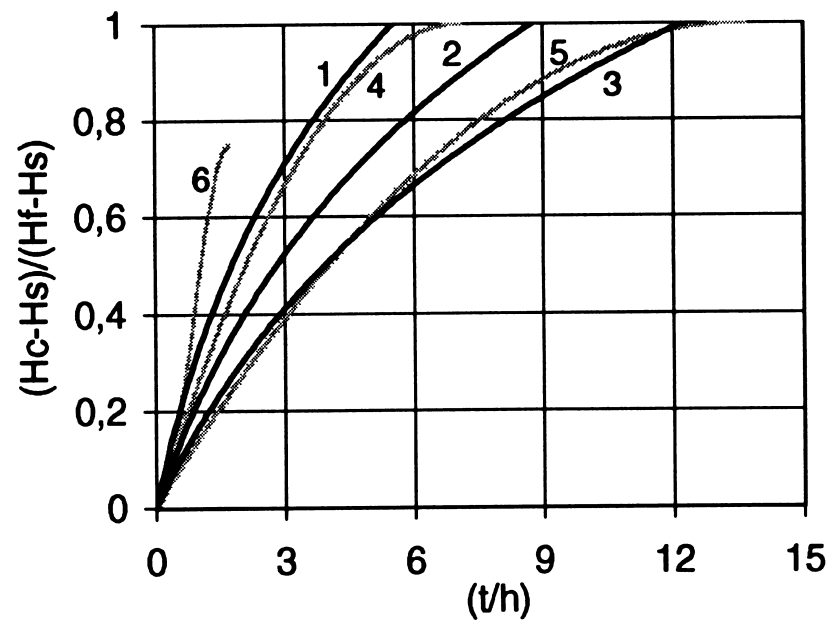

Fig. 1. Results of the present model considering three possibilities: $a=$ $1.574 h$ (curve 1), $a=2.5 h$ (curve 2) and $a=3.5 h$ (curve 3); the value of $(c / a)$ is always equal to 4.5 . Results of other models: area mixture model with $C=0.073$ (curve 4) and $C=0.140$ (curve 5) $[1,3]$ and volume mixture model (curve 6) [5,7].

viour during the hardness test, the first problem is to work out the correspondence between the inner radius $a$ and the dimension of the indentation $h$. Two hypothesis limits can be considered. The first one is to consider $a$ as the radius of the indentation at the surface of the sample: $a=D / 2=3.5 h$ [21] ( $D$ being the diagonal of the indentation and $h$ the indentation depth: $D=7 h)$. The other one considers the removed volume by the indentation to be the same in both cases, i.e. pyramidal indenter and spherical cavity [8,22]. For this hypothesis: $a=1.574 h$. Probably, the best approach is somewhere between these limit values. Fig. 1 shows the results of Eq. (28) considering three relationships between $a$ and h: $a=1.574 h, a=2.5 h$ and $a=3.5 h$ (the value of $(c / a)$ was considered equal to 4.5 - see discussion below). For comparison, this figure also shows the results

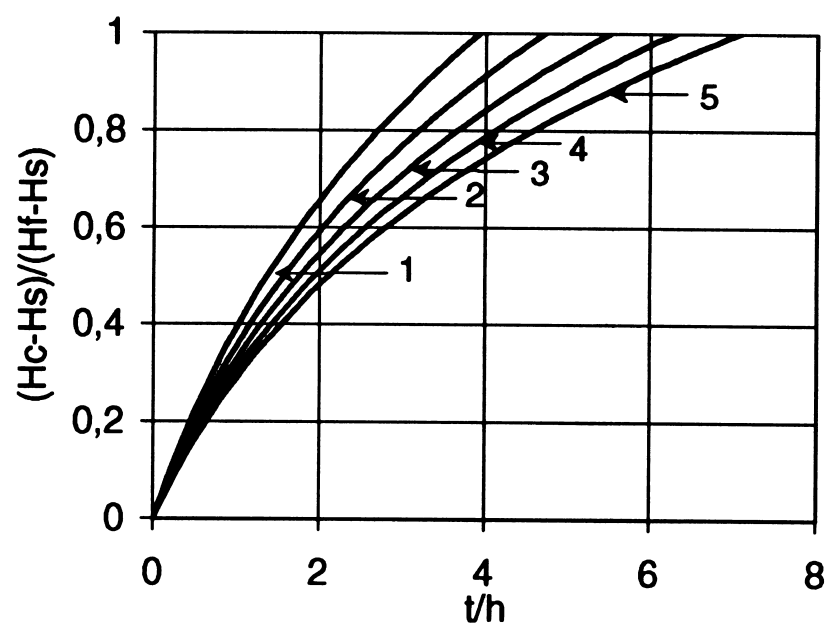

Fig. 2. Influence of $(c / a)$ on the present model results, in accordance with Eq. (28). Curve 1: $c / a=3.5$; curve $2: c / a=4$; curve 3 : $c / a=4.5$; curve 4: $c / a=5$; curve $5: c / a=5.5$. The value of $a$ was considered equal to 1.574 . 


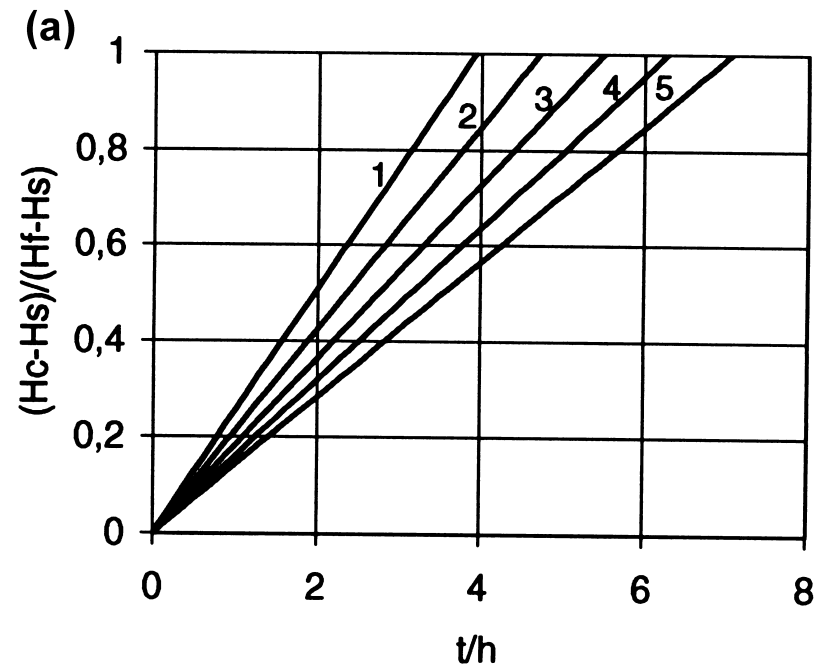

(b)

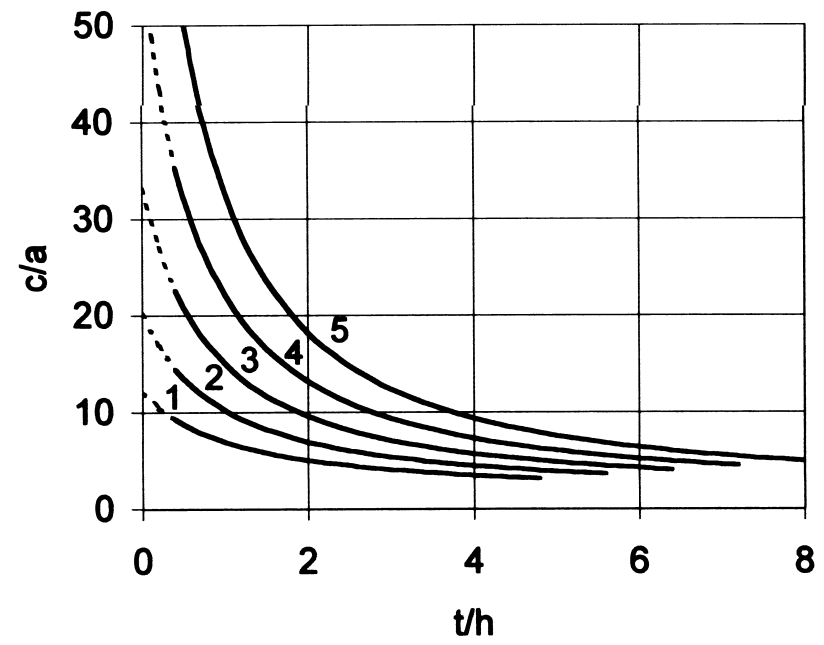

Fig. 3. (a) Linear evolution of $\left(H_{\mathrm{c}}-H_{\mathrm{s}}\right) /\left(H_{\mathrm{f}}-H_{\mathrm{s}}\right)$ versus $(t / h)$; (b) correspondent evolution of $(c / a)$ with $(t / h)$ in agreement with Eq. (31). The value of $a$ was considered equal to 1.574 . The examples exhibit the same $(t / h)_{c}$ as that in Fig. 2.

predicted by some other coated surface hardness models. As can be observed, the results are very sparse. It is not easy to choose the appropriate model to be applied in each case. In most of the cases, none of these models can be successfully applied. Two main kinds of problems are related with the use of the models: the values of the respective empirical parameters may be not correctly defined and some elastic and plastic properties of the film and substrate materials, such as Young's modulus, yield stress, strain hardening rate, etc. are not known a priori and, consequently, the models are not accurate enough or useful.

As mentioned above, the results of the kinematic model are comparable to the ones of some other models previously proposed. Consequently, we do not intend to undertake an experimental validation of the present model. It allows us to perform a qualitative study concerning several mechanical parameters of the film and the substrate. Moreover, it makes it possible to understand the reasons why the models could

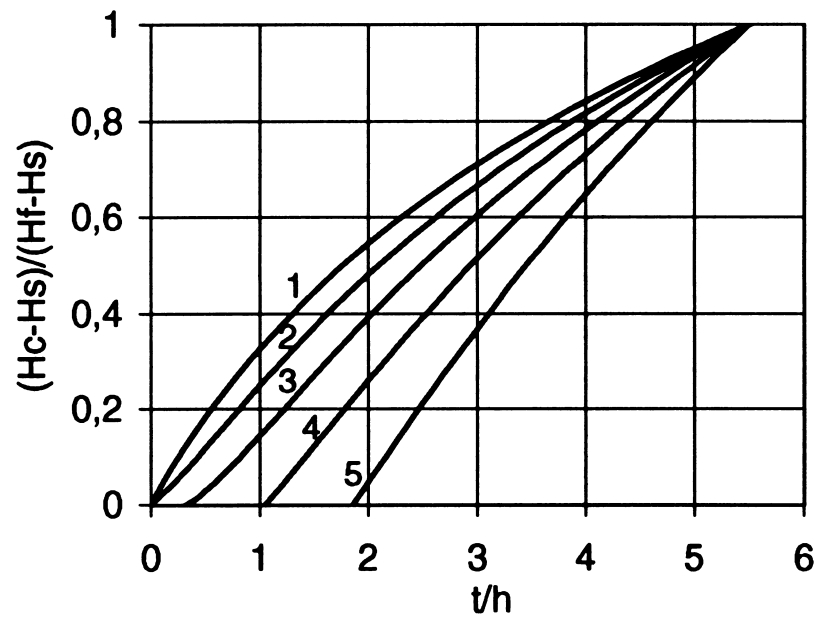

Fig. 4. Importance of the ratio $\alpha_{s} /\left(\Sigma_{0}-\sigma_{0}\right)$. Curve $1 \alpha_{s} /\left(\Sigma_{0}-\sigma_{0}\right)=0$; curve $2 \alpha_{s} /\left(\Sigma_{0}-\sigma_{0}\right)=1$; $\quad$ curve $3 \alpha_{s} /\left(\Sigma_{0}-\sigma_{0}\right)=2$; curve 4 $\alpha_{s} /\left(\Sigma_{0}-\sigma_{0}\right)=3$; curve $5 \alpha_{s} /\left(\Sigma_{0}-\sigma_{0}\right)=4$. The value of $a$ was considered equal to $1.574, c / a=4.5$ and $x=0$.

fail in predicting the hardness of the film, allowing a better understanding of the range of applicability of such models. The examples next studied consider the value of $a$ complying to the relationship $a=1.574 h$. This choice corresponds to the value more currently used in the literature $[8,22]$.

The first study concerns the importance of the value of the ratio $(c / a)$ on the behaviour described by Eq. (1). Neglecting the work-hardening ( $\alpha=0$ ), Eq. (19) allows us to determine the value of $(c / a)$ for any material: $(c / a)=\exp \left(H V / 2 \sigma_{0}\right)$. For most of the experimental cases the ratio $\left(H V / \sigma_{0}\right)$ is close to $3[8,20]$. So, the value of $(c / a)$ must be close to $\exp (1.5) \approx 4.5$. Several attempts were made to evaluate the relative size of the deformed region [21-27]. Also, Bishop et al. [18] have studied this problem, in their original elastoplastic formulation of a spherical hole submitted to internal pressure and have concluded that the ratio $(c / a)$ depends on elastic and plastic properties, particularly on the values of the elastic constants, Young's modulus $(E)$ and Poisson ratio $(\nu)$, and on the yield stress $\left(\sigma_{0}\right)$

$\left(\frac{c}{a}\right)\left[\frac{E}{(1+\nu) \sigma_{0}}\right]^{1 / 3}$

When $\left(E / \sigma_{0}\right)$ is greater than or equal to about 100 , the value of $(c / a)$ is greater than or equal to about 4.25 , depending on the value of $(\nu)$. This hypothesis justifies the use of the rigid-plastic behaviour and considers most of the results in the literature, even for some ceramic materials.

The examples now studied considers Eq. (28), for values of the ratio ( $c / a)$ between 4.0 and 6.0, as shown in Fig. 2. This figure shows that the value of $(t / h)_{c}$, for which $\left(H_{\mathrm{c}}-H_{\mathrm{s}}\right) /\left(H_{\mathrm{f}}-H_{\mathrm{s}}\right)=1$, is strongly influenced by the value of $(c / a)$ (which characterises the size of the deformed region): the bigger this region the higher the value of $(t / h)_{c}$. This agrees with the analysis presented by the authors elsewhere [13]. However, the linear behaviour used to describe 


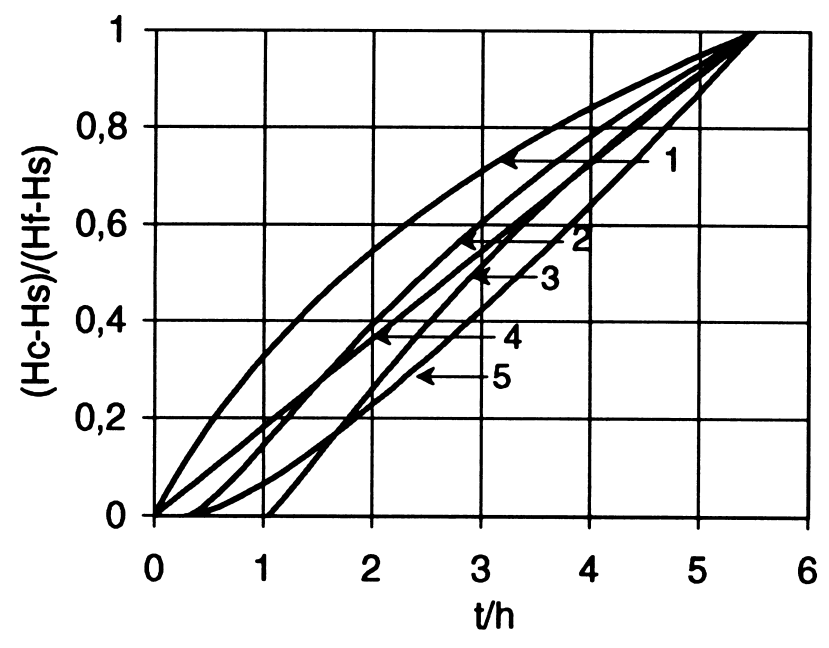

Fig. 5. Comparison between different cases for the model constants. Curve 1: $\alpha_{s} /\left(\Sigma_{0}-\sigma_{0}\right)=0$ and $c / a=4.5$; curve $2: \alpha_{s} /\left(\Sigma_{0}-\sigma_{0}\right)=2$ and $c / a=4.5$; curve 3: $\alpha_{s} /\left(\Sigma_{0}-\sigma_{0}\right)=3$ and $c / a=4.5$; curve $4: \alpha_{s} /\left(\Sigma_{0}-\right.$ $\left.\sigma_{0}\right)=0$ and $(c / a)$ defined by Eq. (31); curve 5: $\alpha_{s} /\left(\Sigma_{0}-\sigma_{0}\right)=2$ and (c/a) defined by Eq. (31). For all the cases, the value of $a$ was considered equal to 1.574 and $x=0$.

the function $\left(H_{\mathrm{c}}-H_{\mathrm{s}}\right) /\left(H_{\mathrm{f}}-H_{\mathrm{s}}\right)$ versus $(t / h)$, experimentally observed, has no correspondence with the results of the model, presented in Figs. 1 and 2. This is probably due to the fact that the hardness test is not quite well simulated by the cavity model. Besides, one must take into account that the ratio $(c / a)$ evolves during the test, because the mechanical behaviour of the material under the indentation changes during the test. In fact, at the beginning of the indentation, the plastic deformation affects only the film and, with increasing penetration depth, the plastic deformation affects also the substrate, in an increasing manner. After the results of Bishop et al. [18] mentioned above in Eq. (30), the size of the deformed region is greater for soft materials, such as those usually used for substrate, than for hard materials, such as those often used for the films (the value of the yield stress should decrease relatively quicker than the value of the Young's modulus and so $(c / a)$ should increase during the hardness test). So, with increasing penetration depth, the value of $(c / a)$ increases, which means that the function $\left(H_{c}-H_{\mathrm{s}}\right) /\left(H_{\mathrm{f}}-H_{\mathrm{s}}\right)=\mathrm{f}(t / h)$ tends to approach linearity. The linearity occurs when $\ln (c / a)$ evolves with $(t / h)$ in such a way that

$\ln \left(\frac{c}{a}\right)=\left(\frac{t}{h}\right)_{c}\left(\frac{t}{h}\right)^{-1} \ln \left(1+\frac{t}{a}\right)$

Under these conditions, Eq. (28) becomes linear

$\frac{H_{\mathrm{c}}-H_{\mathrm{s}}}{H_{\mathrm{f}}-H_{\mathrm{s}}}=\left(\frac{t}{h}\right)_{c}^{-1}\left(\frac{t}{h}\right)$

Fig. 3a shows examples of the function described by the Eq. (32). The corresponding evolution of $(c / a)$ versus $(t / h)$, in accordance with Eq. (31), is shown in Fig. 3b. An important conclusion can already be emphasised: the size of the plastic deformed region and its evolution during the hardness test strongly influence the composite behaviour.

In spite of the impossibility to correctly define this evolution, this model is quite helpful for understanding which parameters define the composite behaviour. Other parameters can be analysed, such as the ratio $k$ between the yield stresses of the film and the substrate. Neglecting the work-hardening of the substrate $\left(\alpha_{\mathrm{s}}=0\right)$, the functions described by Eqs. (26) and (27) are not affected by the ratio $k$ of the yield stresses, giving place to Eqs. (28) and (29). When the work-hardening of the substrate $\alpha_{\mathrm{s}}$ is different from zero, the behaviour is defined by the ratio $\alpha_{\mathrm{s}} /\left(\Sigma_{0}-\right.$ $\sigma_{0}$ ) (see Eq. (27)). An example of such a study is shown in Fig. 4. In this figure, several cases with different values for the ratio $\alpha_{\mathrm{s}} /\left(\Sigma_{0}-\sigma_{0}\right)$ are compared. When this ratio is increased, a region with positive concavity is observed, for values of $(t / h)$ close to zero, i.e. the curve described by Eqs. (27) or (28) is pushed down and to the right, at
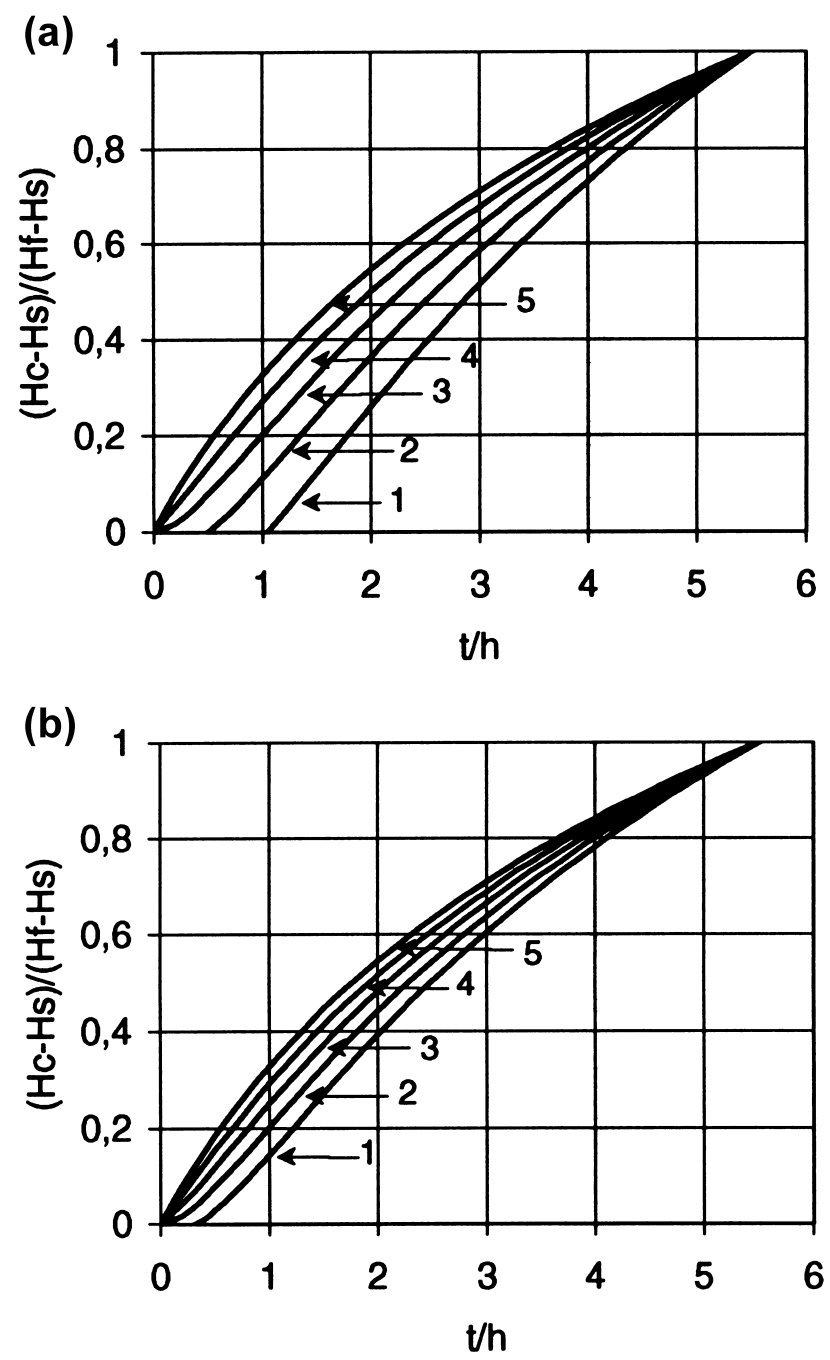

Fig. 6. Influence of the value of $x$ on the model results. Curve 1: $x=0$; curve 2: $x=0.25$; curve $3: x=0.5$; curve $4: x=0.75$; curve $5: x=1$. The value of $a$ was considered equal to 1.574 and $c / a=4.5$ with: (a) $\alpha_{s} /\left(\Sigma_{0}-\right.$ $\left.\sigma_{0}\right)=3$ and (b) $\alpha_{s} /\left(\Sigma_{0}-\sigma_{0}\right)=2$. 
the origin of the axis. At this point, the slope of the curve can even be zero or negative. In the case of $x=0$, this happens for:

$\left(\frac{\alpha_{\mathrm{s}}}{\Sigma_{0}-\sigma_{0}}\right) \geq \frac{3}{2}$

When this occurs, the curve crosses the horizontal axis at two points, $(t / h)=0$ and $(t / h)>0$, in the domain $0 \leq\left(H_{\mathrm{c}}-H_{\mathrm{s}}\right) /\left(H_{f}-H_{\mathrm{s}}\right) \leq 1$. So, the effect of increasing the ratio $\alpha_{\mathrm{s}} /\left(\Sigma_{0}-\sigma_{0}\right)$ is, in some way, qualitatively similar to the effect obtained by increasing the value of $(c / a)$, as was described in Fig. 3a. Increasing both parameters simultaneously, it is possible to observe a positive concavity throughout the domain: $0 \leq\left(H_{\mathrm{c}}-H_{\mathrm{s}}\right) /\left(H_{\mathrm{f}}-H_{\mathrm{s}}\right) \leq 1$. In Fig. 5, several different cases are considered for comparison. The above mentioned effect is clearly seen when we observe curve 4 and 5: curve 4 was obtained with a constant value of $(c / a)$ and a large value of $\alpha_{\mathrm{s}} /\left(\Sigma_{0}-\sigma_{0}\right)$ (a positive concavity is observed only for values of (t/h) close to zero) while curve 5 is obtained with the same value of $\alpha_{\mathrm{s}} /\left(\Sigma_{0}-\right.$ $\left.\sigma_{0}\right)$ but with $(c / a)$ evolving with $(t / h)$ in agreement with Eq. (31) (the concavity is positive in the entire range of values of $(t / h))$.

It must be referred that, in the present paper, the cavity problem is intended to be solved using the kinematic model for the case of a hard film on a relatively softer substrate: $\left(H_{\mathrm{f}} / H_{\mathrm{s}}\right)>1$. Under this hypothesis, the value of $\alpha_{\mathrm{s}} /\left(\Sigma_{0}-\right.$ $\sigma_{0}$ ) obeys the condition as follows (whatever the value of $x \geq 0$ ), as in the examples shown in Figs. 4 and 5

$\left(\frac{\alpha_{\mathrm{s}}}{\Sigma_{0}-\sigma_{0}}\right)<\frac{9}{2} \ln \left(\frac{c}{a}\right)$

It can be concluded that the results are influenced by the ratio $\alpha_{\mathrm{s}} /\left(\Sigma_{0}-\sigma_{0}\right)$, mainly for low values of $(t / h)$. Also, the fact that the film presents a work-hardening coefficient different from zero changes the behaviour for low $(t / h)$ values, as seen in Fig. 6, for which the $x$ value changes between 0 and 1 . Whatever the case of $\alpha_{\mathrm{s}} /\left(\Sigma_{0}-\sigma_{0}\right)$ and $x,(t / h)_{c}$ it only depends on the value that takes $(c / a)$.

\section{Conclusions}

The kinematic model was applied to the case of a spherical cavity problem, in order to simulate the mechanical behaviour of a coated material during the hardness test. It can be concluded that the present formulation as well as the case of other former models do not give any guarantee of accuracy in the solving of such a problem. This guarantee fails mainly concerning the definition of the values of the parameters of the models, which are sometimes empirical and at other times dependant on the properties of the film and the substrate. Most of the times, the correct values of the parameters are not known. The present model allows a qualitative study concerning the parameters that determine the behaviour of the composite film-substrate, when both deform plastically. The results of the model show that an important parameter that influences this behaviour is the size of the plastically deformed region, which mainly influences the behaviour for relatively high values of $(t / h)$. The film and the substrate properties also influence the behaviour of the composite, this time for low $(t / h)$ values.

\section{Acknowledgements}

The authors are indebted to JNICT for financial support through FEDER and Program PRAXIS XXI.

\section{References}

[1] B. Jönsson, O. Hogmark, Thin Solid Films 114 (1984) 257.

[2] P.J. Burnett, T.F. Page, J. Mater. Sci. 19 (1984) 851.

[3] O. Vingsbo, S. Hogmark, B. Jönsson, A. Ingemarsson, in: P.J. Blau, B.R. Lawn (Eds.), Microindentation Techniques in Materials Science and Engineering, ASTM Special Technical Publication, Vol. 889, American Society for Testing and Materials, Philadelphia, PA, 1985 , p. 257.

[4] A. Thomas, Surf. Eng. 3 (1987) 117.

[5] P.J. Burnett, D.S. Rickerby, Thin Solid Films 148 (1987) 41.

[6] P.J. Burnett, D.S. Rickerby, Thin Solid Films 148 (1987) 51

[7] P.J. Burnett, D.S. Rickerby, Surf. Eng. 3 (1987) 69.

[8] I.J. Ford, Thin Solid Films 245 (1994) 122.

[9] D. Chicot, J. Lesage, Thin Solid Films 254 (1995) 123.

[10] N.G. Chechenin, J. Bøtigger, J.P. Krog, Thin Solid Films 261 (1995) 219.

[11] M. Witlling, A. Bendavid, P.J. Martin, M.V. Swain, Thin Solid Films 2270 (1994) 283.

[12] J. Manika, J. Maniks, Thin Solid Films 2208 (1992) 227.

[13] J.V. Fernandes, A.C. Trindade, L.F. Menezes, J. Mater. Res. (1998), submitted.

[14] C.R. Boër, N. Rebelo, H. Rydstad, G. Schröder, Process Modelling of Metal Forming and Thermomechanical Treatment, Springer-Verlag, Berlin, 1986.

[15] R. Hill, The Mathematical Theory of Plasticity, Clarendon, Oxford, 1950.

[16] D. Lebouvier, P. Gilormini, E. Felder, J. Phys. D Appl. Phys. 18 (1985) 119.

[17] D. Lebouvier, P. Gilormini, E. Felder, Thin Solid Films 172 (1989) 227.

[18] R.F. Bishop, R. Hill, N.F. Mott, Proc. Phys. Soc. 57 (1945) 147.

[19] T.O. Mulhearn, J. Mech. Phys. Solids 18 (1959) 85.

[20] D. Tabor, in: P.J. Blau, B.R. Lawn (Eds.), Microindentation Techniques in Materials Science and Engineering, ASTM Special Technical Publication, Vol. 889, American Society for Testing and Materials, Philadelphia, PA, 1985, p. 129.

[21] K.L. Johnson, J. Mech. Phys. Solids 18 (1970) 115.

[22] S.S. Chiang, D.B. Marshall, A.G. Evans, J. Appl. Phys. 53 (1982) 298.

[23] A.G. Evans, E.A. Charles, J. Am. Ceram. Soc. 59 (1976) 371.

[24] B.R. Lawn, A.G. Evans, D.B. Marshall, J. Am. Ceram. Soc. 63 (1980) 574.

[25] M. Yoshida, J. Appl. Phys. 76 (1994) 7790.

[26] M.T. Laugier, J. Mater. Sci. Lett. 4 (1995) 1539.

[27] W. Yu, J.P. Blanchard, J. Mater. Res. 11 (1996) 2358. 